\title{
STBC MC-CDMA Systems for Indoor and Outdoor Scenarios
}

\author{
F. Portier, J-F. Hélard, J-M. Auffray, J-Y. Baudais \\ IETR, UMR CNRS 6164 \\ INSA, 20 avenue des Buttes-de-Coësmes, CS 14215, 35043 Rennes Cedex, FRANCE \\ Email: \{fabrice.portier, jean-francois.helard, jean-michel.auffray, jean-yves.baudais\}@insa-rennes.fr
}

\begin{abstract}
In this paper, we compare the performance of Alamouti's space-time block coded MC-CDMA systems for indoor and outdoor realistic scenarios with zero forcing or minimum mean square error detection schemes. Two different configurations of the system are considered for the two scenarios. The different results obtained as well for indoor as for outdoor scenarios demonstrate that spatial diversity improves significantly the performance of MC-CDMA systems. Then, Alamouti's STBC MC-CDMA schemes derive full benefit from the frequency and spatial diversities and can be considered as a very realistic and promising candidate for the air interface downlink of the 4th generation mobile radio systems.
\end{abstract}

\section{INTRODUCTION}

The Multi-Carrier Code Division Multiple Access (MCCDMA) modulation scheme has already proven to be a strong candidate as an access technique for broadband cellular systems [1]. Different concepts based on the combination of multi-carrier modulation with direct sequence code division multiple access have been introduced in 1993 [2], [3], [4], [5]. Since that time, owing to its high spectral efficiency and high flexibility, MC-CDMA is considered as a very promising technique, specifically for the downlink of future cellular mobile radio systems. Indeed, MC-CDMA combines the robustness of Orthogonal Frequency Division Multiplex (OFDM) with the flexibility of CDMA [6], [7]. Thus, MC-CDMA is for example studied within the European IST project MATRICE. This work has been partly carried out within this project which aims at defining a new air interface for $4 \mathrm{G}$ systems.

On the other hand, Multiple Input Multiple Output (MIMO) communication systems, by using several antennas, inherit space diversity to mitigate fading effects. When the channel is not known at the transmitter, taking advantage of the transmit diversity requires methods such as space-time coding which uses coding across antennas and time. For example, SpaceTime Block Coding (STBC), as proposed by Alamouti in [8] and Tarokh in [9], provides full spatial diversity gains, no intersymbol interference and low complexity maximum likelihood receiver if transmission matrix is orthogonal. Moreover with STBC, only one receive antenna can be used, leading in that case to Multiple Input Single Output (MISO) systems. In that design of two transmit antennas and one receive antenna, Alamouti's STBC is also optimal for capacity.

In [10], it has been shown that unity-rate Alamouti's STBC QPSK MC-CDMA outperforms half-rate Tarokh's STBC 16QAM MC-CDMA over theoretical Rayleigh channel, while offering the same effective throughput of $2 \mathrm{bit} / \mathrm{s} / \mathrm{Hz}$ without channel coding. Indeed, in order to maintain the same effective throughput, half-rate STBC have to be employed with higher modulation schemes as 16-QAM, which are more prone to errors and hence degrade the performance of the system.

In this paper, we compare the performance of Alamouti's STBC MC-CDMA systems for indoor and outdoor applications with Zero Forcing (ZF) or Minimum Mean Square Error (MMSE) Single-user Detection (SD) schemes. Two different MC-CDMA systems have been considered for the two scenarios. The first proposed configuration, which parameters set is based on HIPERLAN Type 2 specifications, is evaluated for indoor propagation scenarios using the realistic stochastic MIMO channel model developed within the European IST project METRA (Multi Element Transmit Receive Antennas). The second proposed configuration, based on a 57.6 MHz sampling frequency, is tested for outdoor propagation scenarios using a 3GPP2-like MIMO channel model.

This paper is organized as follows. Section 2 describes the studied orthogonal STBC MC-CDMA system. Section 3 deals with the choice of the system parameters for the two scenarios. Section 4 describes the METRA channel model and gives the performance of STBC MC-CDMA systems with ZF and MMSE $\mathrm{SD}$ for indoor propagation scenarios. Section 5 presents the 3GPP2-like MIMO channel model and additional results are given with and without channel coding for outdoor propagation scenarios. Finally, Section 6 summarizes results and concludes the paper.

\section{SYSTEM DESCRIPTION}

\section{A. General Presentation}

Figure 1 shows the considered MIMO MC-CDMA system for the $j$ th user based on Alamouti's STBC with $N_{t}=2$ transmit antennas and $N_{r}=2$ receive antennas. Each user $j$ transmits simultaneously from the two antennas the symbols $x_{j}^{0}$ and $x_{j}^{1}$ at time $u$, and the symbols $-x_{j}^{1 *}$ and $x_{j}^{0 *}$ at time $u+T_{x}$ where $T_{x}$ is the OFDM symbol duration. At the output of the spacetime encoder, the data symbols $x^{0}=\left[x_{1}^{0} \cdots x_{j}^{0} \cdots x_{N_{u}}^{0}\right]^{T}$ of the $N_{u}$ users are multiplied by their specific orthogonal WalshHadamard spreading code $c_{j}=\left[c_{j, 1} \cdots c_{j, L_{c}}\right]^{T}$ where $c_{j, k}$ is the $k$ th chip, and $[\cdot]^{T}$ denotes matrix transposition (the same goes for symbol $\left.\boldsymbol{x}^{1}\right) . \boldsymbol{c}_{j}$ is the $j$ th column vector of the $L_{c} \times N_{u}$ spreading code real-valued matrix $\boldsymbol{C}$. In this paper, the length $L_{c}$ of the spreading sequences is equal to the 
number $N_{c}$ of subcarriers for indoor scenarios. For outdoor scenarios, as $L_{c} \leq N_{c}$, a linear frequency multiplexing is carried out. In the following equations, we consider only $L_{c}$ subcarriers without losing generality as the extension to $N_{c}=\alpha \cdot L_{c}$ subcarriers is straightforward. Each data symbol is then transmitted in parallel on $L_{c}$ QPSK modulated subcarriers. The vector obtained at the $r$ th receive antenna after the OFDM demodulation and deinterleaving, at time $u$ and $u+T_{x}$, is given by:

$$
\boldsymbol{R}_{r}=\mathcal{H}_{r} \mathcal{C X}+\boldsymbol{N}_{r}, \text { with } \mathcal{H}_{r}=\left[\begin{array}{cc}
\boldsymbol{H}_{1 r} & \boldsymbol{H}_{2 r} \\
\boldsymbol{H}_{2 r}^{*} & -\boldsymbol{H}_{1 r}^{*}
\end{array}\right]
$$

where $\boldsymbol{R}_{r}=\left[\boldsymbol{r}_{r}^{T}(u) \boldsymbol{r}_{r}^{H}\left(u+T_{x}\right)\right]^{T}$ with $\boldsymbol{r}_{r}(u)=\left[r_{r, 1}(u)\right.$ $\left.\cdots r_{r, L_{c}}(u)\right]^{T}$ the vector of the $L_{c}$ received signals at time $u$ and [. $]^{H}$ denotes the Hermitian (or complex conjugate transpose), $\boldsymbol{H}_{t r}=\operatorname{diag}\left(h_{t r, 1}, \cdots, h_{t r, L_{c}}\right)(t, r \in\{1,2\})$ is a $L_{c} \times L_{c}$ diagonal matrix with $h_{t r, k}$ the complex channel frequency response, for the subcarrier $k$ from the transmit antenna $t$ to the receive antenna $r$. Time invariance during two MC-CDMA symbols is assumed to permit the recombination of symbols when STBC is used. $\mathcal{C}=\operatorname{diag}(\boldsymbol{C}, \boldsymbol{C})$ where $\boldsymbol{C}=$ $\left[\boldsymbol{c}_{1} \cdots \boldsymbol{c}_{j} \cdots \boldsymbol{c}_{N_{u}}\right]$ is the $L_{c} \times N_{u}$ matrix of user's spreading codes, and $\boldsymbol{X}=\left[\begin{array}{ll}\boldsymbol{x}^{0 T} & \boldsymbol{x}^{1 T}\end{array}\right]^{T} . \boldsymbol{N}_{r}=\left[\boldsymbol{n}_{r}^{T}(u) \boldsymbol{n}_{r}^{H}\left(u+T_{x}\right)\right]^{T}$ where $\boldsymbol{n}_{r}(u)=\left[n_{r, 1}(u) \cdots n_{r, L_{c}}(u)\right]^{T}$ is the Additive White Gaussian Noise vector with $n_{r, k}(u)$ representing the noise term at subcarrier $k$, for the $r$ th receive antenna at time $u$ with variance given by $\sigma_{k}^{2}=\mathrm{E}\left[\left|n_{k}\right|^{2}\right]=N_{0} \forall k$.
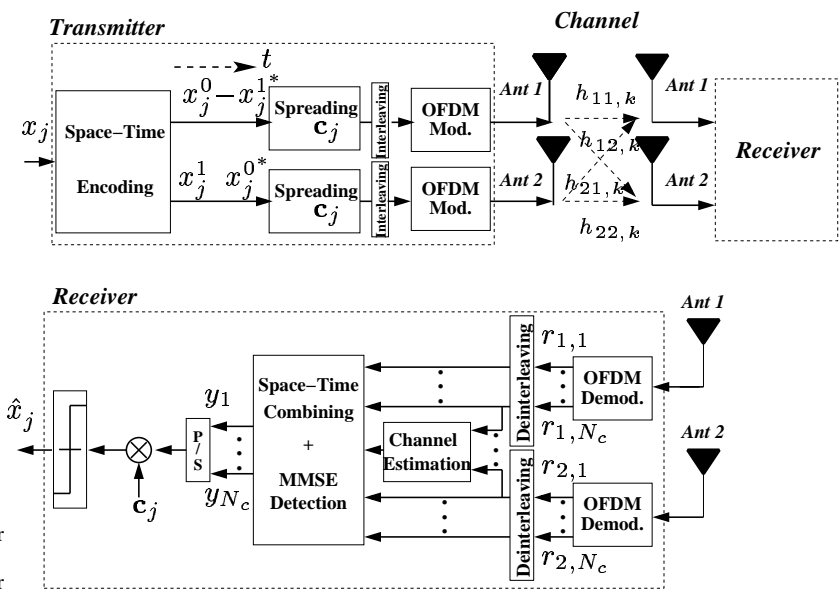

Fig. 1. MC-CDMA transmitter and receiver for user $j$ with transmit and receive diversities.

In the receiver, in order to detect the two transmitted symbols $x_{j}^{0}$ and $x_{j}^{1}$ for the desired user $j$, ZF or MMSE SD detection schemes are applied to the received signals in conjunction with STBC decoding. In the SISO case, MMSE SD is the most efficient linear SD scheme [7]. In this paper, in order to evaluate the gain of MISO and MIMO systems, the performance of ZF and MMSE detection with SISO schemes are given as reference.

\section{B. MMSE and ZF Single-user Detection in MISO or MIMO}

After equalization, for each receive antenna $r$, the two successive received signals are combined. The resulting signals from the $N_{r}$ receive antennas are then added to detect the two symbols $x_{j}^{0}$ and $x_{j}^{1}$. After despreading and threshold detection, the detected data symbols $\hat{x_{j}^{0}}$ and $\hat{x_{j}^{1}}$ for user $j$ are:

$$
\begin{aligned}
{\left[\begin{array}{ll}
\hat{x}_{j}^{0} & \hat{x}_{j}^{1}
\end{array}\right]^{T}=} & \left(\boldsymbol{I}_{2} \otimes \boldsymbol{c}_{j}^{T}\right) \boldsymbol{Y}=\left(\boldsymbol{I}_{2} \otimes \boldsymbol{c}_{j}^{T}\right) \sum_{r=1}^{N_{r}} \mathcal{G}_{r} \boldsymbol{R}_{r} \\
& \text { with } \mathcal{G}_{r}=\left[\begin{array}{cc}
\boldsymbol{G}_{1 r} & \boldsymbol{G}_{2 r}^{*} \\
\boldsymbol{G}_{2 r} & -\boldsymbol{G}_{1 r}^{*}
\end{array}\right]
\end{aligned}
$$

where $\boldsymbol{I}_{2}$ is the $2 \times 2$ identity matrix, $\otimes$ is the Kronecker product, $\boldsymbol{Y}=\left[\begin{array}{lll}y_{1}^{0} \cdots y_{L_{c}}^{0} & y_{1}^{1} \cdots y_{k}^{1} \cdots y_{L_{c}}^{1}\end{array}\right]^{T}$ is the vector of the received signals equalized and combined from the $N_{r}$ antennas. $\boldsymbol{G}_{t r}$ is a diagonal matrix (as we use an SD scheme) containing the equalization coefficients for the channel between the transmit antenna $t$ and the receive antenna $r$. To detect for example $x_{i}^{0}$, the MMSE SD coefficients $g_{t r, k}$ minimize the mean square value of the error $\varepsilon_{k}^{0}$ between the signal $\sum_{i=1}^{N_{u}} c_{i, k} x_{i}^{0}$ transmitted on subcarrier $k$ and the received signals combined from the $N_{r}$ receive antennas by the Alamouti's decoding. In the same way, the ZF coefficients $g_{t r, k}$ restore the orthogonality between the different users. It is well known that with SISO systems, ZF leads to excessive noise amplification for low subcarrier Signal to Noise Ratio (SNR). But in the MIMO case, due to the spatial diversity, which is here equal to the product $N_{t} \times N_{r}$ in the decorrelated situation, this occurrence is statistically reduced. Thus, with an increasing number of antennas, ZF tends to MMSE efficiency, and does not require a SNR estimation of $\gamma$ at receiver. We assume the same noise level statistically whatever the subcarrier or receive antenna. Besides, no knowledge of the spreading codes $\boldsymbol{c}_{i}(i \neq j)$ of the interfering users is required to derive the ZF and MMSE single-user detection coefficients.

TABLE I

ZF AND MMSE EQUALIZATION COEFFICIENTS $g_{t r, k}$ AND RESULTING EQUALIZED CHANNEL TERMS TO DETECT THE SYMBOL $x_{j}^{0}$.

\begin{tabular}{|c|c|c|}
\hline & ZF & MMSE \\
\hline$g_{t r, k}$ & $\frac{h_{t r, k}^{*}}{\sum_{t} N_{r}}\left|h_{t r, k}\right|^{2}$ & $\frac{h_{t r, k}^{*}}{\sum_{t=1}^{N_{t}} \sum_{r=1}^{N_{r}}\left|h_{t r, k}\right|^{2}+\frac{1}{\gamma}}$ \\
\hline$h_{e q, k}^{0}$ & 1 & $\beta=\frac{\sum_{t=1}^{N_{t}} \sum_{r=1}^{N_{r}}\left|h_{t r, k}\right|^{2}}{\sum_{t=1}^{N_{t}} \sum_{r=1}^{N_{r}}\left|h_{t r, k}\right|^{2}+\frac{1}{\gamma}}$ \\
\hline$h_{e q, k}^{1}$ & 0 & 0 \\
\hline
\end{tabular}

Table I gives the ZF and MMSE equalization coefficients $g_{t r, k}$ and the resulting equalized channel coefficients $h_{e q, k}^{0}$ and $h_{e q, k}^{1}$ to detect $x_{i}^{0}$ and to cancel $x_{i}^{1}$ respectively. For the two algorithms based on orthogonal STBC per subcarrier, $N_{t} \times N_{r}$ channel coefficients $h_{t r, k}$ are taken into account, decreasing 
the probability of an excessive noise amplification for low subcarrier SNR. In both cases, to detect for example $x_{i}^{0}$, the interference terms generated by $x_{i}^{1}$ are canceled, i.e. $h_{e q, k}^{1}=0$. Note that the threshold detection should be normalized by $\beta$ for MMSE with high-order modulations (16QAM, ...).

\section{SYSTEM PARAMETERS}

The system parameters are chosen according to the time and frequency coherence of the channel in order to reduce intersubcarrier interference and Inter-Symbol Interference (ISI). Besides, investigated MC-CDMA configurations are designed to propose high throughput and high capacity solutions for indoor and outdoor scenarios.

TABLE II

MAIN SYSTEM PARAMETERS.

\begin{tabular}{lcc}
\hline \multicolumn{1}{c}{ System parameters } & Configuration I & Configuration II \\
\hline Sampling frequency $F_{s}$ & $20 \mathrm{MHz}$ & $57.6 \mathrm{MHz}$ \\
FFT size & 64 & 1024 \\
Number $N_{c}$ of used sub-carriers & 64 & 736 \\
Guard Interval duration $T_{g}$ & $0.5 \mu \mathrm{s}$ & $3.75 \mu \mathrm{s}$ \\
Total symbol duration & & \\
\multicolumn{1}{c}{$T_{x}=T_{u}+T_{g}$} & $3.7 \mu \mathrm{s}$ & $21.52 \mu \mathrm{s}$ \\
Subcarrier spacing $\Delta_{f}=1 / T_{u}$ & $312 \mathrm{KHz}$ & $56.2 \mathrm{KHz}$ \\
Length $L_{c}$ of spreading codes & 64 & $16-32$ \\
Modulation & $\mathrm{QPSK}$ & $\mathrm{QPSK}$ \\
Center frequency $f_{c}$ & $5.2 \mathrm{GHz}$ & $5.0 \mathrm{GHz}$ \\
Occupied bandwidth & $20 \mathrm{MHz}$ & $41.46 \mathrm{MHz}$ \\
Frame duration / Guard duration & - & $30 T_{s} / 20.8 \mu \mathrm{s}$ \\
\hline
\end{tabular}

For indoor propagation scenarios, we considered the BRAN A channel profile, with a carrier frequency $f_{c}=5.2 \mathrm{GHz}$ and a maximum delay $\tau_{\max }=390 \mathrm{~ns}$. So, the first configuration proposed for indoor scenarios, which parameters set is summed up in table II, is based on HIPERLAN Type 2 specifications with a $20 \mathrm{MHz}$ sampling frequency. The "useful" part $T_{u}$ of the MC-CDMA symbol is equal to 3.2 $\mu$ s, which leads to a sub-carrier spacing equal to $312 \mathrm{KHz}$. According to $\tau_{\max }$ value and in order to avoid ISI, the guard interval duration $T_{g}$ is chosen equal to $0.5 \mu$ s, which leads to a $13 \%$ spectral efficiency loss and a power efficiency loss equal to $0.63 \mathrm{~dB}$. The overall bit-rate is then $34 \mathrm{Mbit} / \mathrm{s}$ for QPSK. The length $L_{c}=64$ of the spreading sequences is equal to the number $N_{c}$ of used subcarriers and to the FFT size.

The second studied configuration proposed for outdoor scenarios is based on a sampling frequency which is a multiple of the $3.84 \mathrm{MHz}$ UMTS frequency, to obtain the same frame duration as UMTS $\left(666 \mu\right.$ s). So $F_{s}$ is equal to $15 \times 3.84=57.6$ $\mathrm{MHz}$. We consider here a channelization bandwidth of 50 $\mathrm{MHz}$, a carrier frequency $f_{c}=5 \mathrm{GHz}$, an FFT size of 1024 with $N_{c}=736$ used sub-carriers. The guard interval duration $T_{g}=3.75 \mu \mathrm{s}$, chosen according to the maximum delay $\tau_{\text {max }}=3.5 \mu \mathrm{s}$, leads to a $18 \%$ spectral efficiency loss and a power efficiency loss equal to $0.84 \mathrm{~dB}$. The overall bit-rate is then $67 \mathrm{Mbit} / \mathrm{s}$ for QPSK without channel coding, shared between users. Furthermore, the length $L_{c}$ of the spreading codes is equal to 16 or 32 , and a linear frequency multiplexing is applied to the symbols before OFDM modulation.
In both scenarios, results are presented assuming perfect channel estimation at receiver, and considering QPSK modulation. For the $E_{b} / N_{0}$ axis of figures, we take into account the system parameters (like the coding rate), except the guard interval efficiency loss. We assume the same total transmit power (whatever the number of transmit antennas), and in the figures we removed the receive antenna gain in the MIMO case, degrading performance of $3 \mathrm{~dB}$.

\section{Simulation RESUltS OVER INDOOR METRA CHANNELS}

A major characteristic of the stochastic MIMO channel model developed within the European research IST METRA project is that, contrary to other directional models, it does not rely on a geometrical description of the environment under study [11]. It is a complex Single-Input Single-Output (SISO) finite impulse response filter whose taps are computed so as to simulate time dispersion, fading and spatial correlation. To simulate MIMO radio channels, it has to be inserted between parallel-to-serial and serial-to-parallel converters. Besides, the correlation properties in the spatial domain of the MIMO radio channel are obtained by the Kronecker product of two independent correlation matrices defining the correlation properties at the Base Station (BS) and Mobile Station (MS).

TABLE III

MAIN MIMO METRA CHANNEL PARAMETERS.

\begin{tabular}{lc}
\hline \multicolumn{1}{c}{ Channel parameters } \\
\hline Channel Profi le & BRAN A \\
Maximum delay $\tau_{\max }$ & $390 \mathrm{~ns}$ \\
Velocity & $3.6 \mathrm{~km} / \mathrm{h}$ \\
Doppler Spectrum & Jakes \\
Pattern & Omnidirectionnel \\
Maximum Doppler frequency & $17.3 \mathrm{~Hz}$ \\
Doppler oversampling factor & 2 \\
Measured coherence bandwidth & $5.8 \mathrm{MHz}$ \\
DoA azimuth & $0^{\circ}$ \\
Elevation angle & $90^{\circ}$ \\
\hline
\end{tabular}

Table III summarizes the main MIMO METRA channel parameters. In the correlated MISO and MIMO cases, we consider 2 antennas at BS and MS with an inter-element separation fixed to $1.5 \lambda$ and $0.4 \lambda$ respectively ( $\lambda$ is wavelength). Then, the envelope correlation coefficients between antennas are:

$$
R_{B S}=\left[\begin{array}{cc}
1 & 0.265 \\
0.265 & 1
\end{array}\right] \quad R_{M S}=\left[\begin{array}{cc}
1 & 0.294 \\
0.294 & 1
\end{array}\right]
$$

which have been derived from $4 \times 4$ correlation matrices obtained through measurements in real indoor scenarios [11].

Figures 2 and 3 represent respectively the performance of ZF and MMSE detection for full load systems $\left(L_{c}=N_{c}=\right.$ $\left.N_{u}=64\right)$ in indoor environment, without channel coding. The performance of the SISO $\left(N_{t} N_{r}=11\right)$ scheme is given as reference. In the MISO $\left(N_{t} N_{r}=21\right)$ and MIMO $\left(N_{t} N_{r}=22\right)$ cases, the $21 \mathrm{ZF}$ and especially the $22 \mathrm{ZF}$ schemes offer good performance. The gains of MISO MMSE and MIMO MMSE is confirmed compared to the SISO MMSE scheme.

Besides, in the realistic case corresponding to correlated channels with a $1.5 \lambda$ and $0.4 \lambda$ separation between the two 


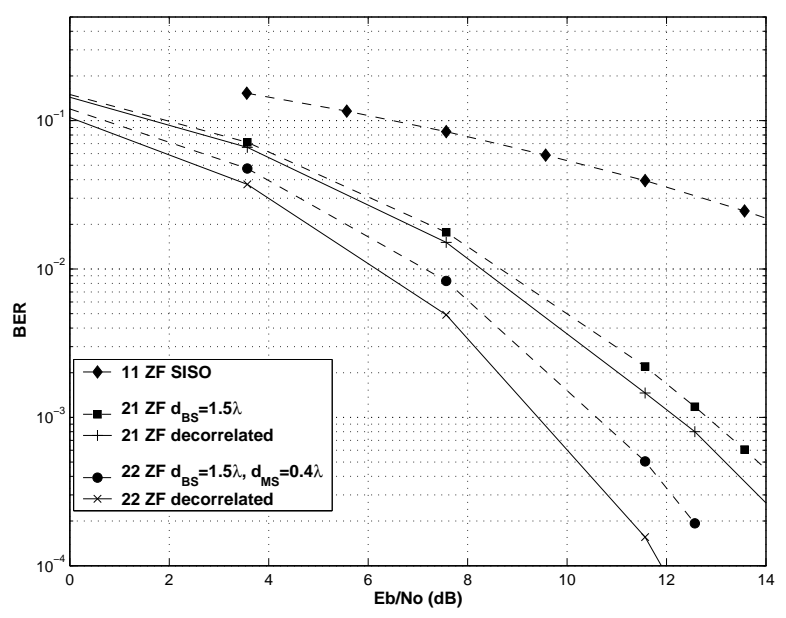

Fig. 2. Performance of ZF $\left(N_{c}=L_{c}=N_{u}=64\right)$ over indoor channels for SISO, MISO and MIMO systems.

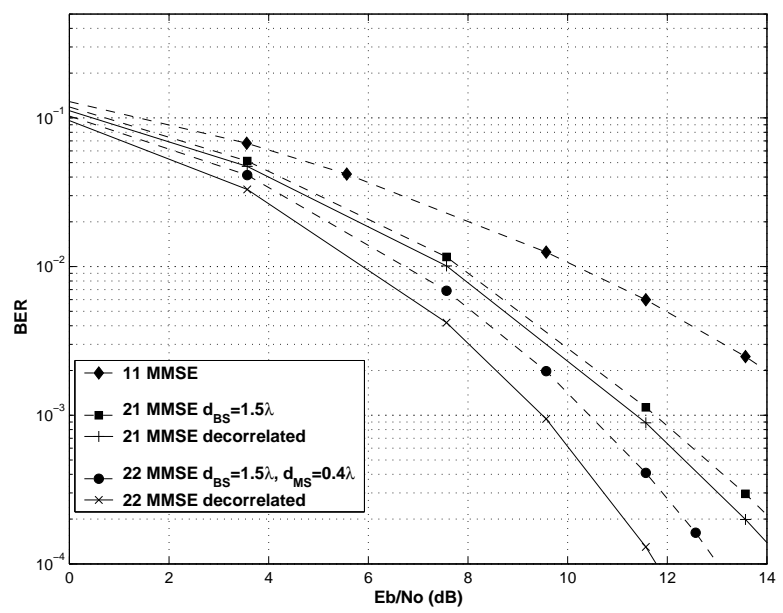

Fig. 3. Performance of MMSE at full load $\left(N_{c}=L_{c}=N_{u}=64\right)$ over indoor channels for SISO, MISO and MIMO systems.

transmit and receive antennas, the performance loss compared to the perfectly decorrelated case is less than $1 \mathrm{~dB}$ for a $\mathrm{BER}=10^{-3}$. These different results demonstrate that in indoor scenarios, characterized by limited frequency and time diversities, exploiting spatial diversity improves significantly the performance.

\section{Simulation Results OVER 3GPP2-Like OUtDoor CHANNELS}

For outdoor propagation scenarios, we used a link level MIMO channel model which has been specifically developed within the European research IST MATRICE project. This channel model is based on the $3 \mathrm{GPP} / 3 \mathrm{GPP} 2$ proposal [12] for a wideband MIMO channel exploiting multipath angular characteristics. It consists in elaborating a spatial model from a hybrid approach between a geometrical concept depending on cluster positions and a tapped delay line model describing the average power delay profile with a fixed number of taps. The spatial parameters are defined at the Base Station (BS) and at the Mobile Station (MS). The angular distribution is modeled from parameters leading to an average angle spread. The model parameters have been adapted at $5 \mathrm{GHz}$ for outdoor environment. Then, the MIMO channel response consists in a sum of sub-rays extracted from the previous statistics.

TABLE IV

MAIN MIMO 3GPP2 CHANNEL PARAMETERS.

\begin{tabular}{lc}
\hline \multicolumn{1}{c}{ Channel parameters } \\
\hline Channel Profi le & BRAN E \\
Maximum delay $\tau_{\max }$ & $1.76 \mu \mathrm{s}$ \\
Number of paths & 17 \\
Number of sub-rays par path & 20 \\
Velocity & $72 \mathrm{~km} / \mathrm{h}$ \\
Mean Angle Spread at BS & $\mathrm{E}\left[\sigma_{\mathrm{AS}}\right]=21.4^{\circ}$ \\
Mean Angle Spread at MS & $\mathrm{E}\left[\sigma_{\mathrm{AS}, \mathrm{MS}}\right]=68^{\circ}$ \\
Mean total RMS Delay Spread & $\mathrm{E}\left[\sigma_{\mathrm{DS}}\right]=0.25 \mu \mathrm{s}$ \\
Element spacing & $0.5 \lambda-10 \lambda$ \\
\hline
\end{tabular}

Table IV summarizes the main MIMO 3 GPP2 channel parameters. We used the BRAN E channel profile to modelize an outdoor urban environment. Outdoor is characterized by a larger delay spread, a higher mobility, and an asymmetrical antenna configuration. The consequent correlation is inferior to 0.1 for $10 \lambda$ (wavelength) spacing, while an antenna spacing of $0.5 \lambda$ leads to a correlation around 0.7 at BS, and 0.35 at MS. Note that this outdoor configuration presents more frequency diversity than the indoor one; the outdoor spatial diversity is however inferior with an antenna spacing of $0.5 \lambda$.

Figure 4 shows the results obtained with $\mathrm{ZF}$ detection and without channel coding. The results do not depend on the number of users with this equalization algorithm, so we ran simulations for full load. As the curves are almost the same for the two considered spreading codes (16 or 32), we only represent those for $L_{c}=N_{u}=32$. This can be explained by the frequency diversity which is inferior to 32 for the BRAN E channel over the considered bandwidth, and mainly exploited with a spreading length of 16 combined combined with linear frequency multiplexing. The gain of MISO is confirmed compared to SISO scheme, even in the most correlated case. Figure 5 shows equivalent results with MMSE detection, leading to the same conclusions. However, when the number of antennas increases, as stated by equations, ZF tends to MMSE performance.

Figure 6 shows the results obtained in a realistic configuration with or without turbo coding. We choose an antenna spacing of $10 \lambda$ at BS and $0.5 \lambda$ at MS, and MMSE detection. The channel coding scheme is the turbo code defined for UMTS, with a rate of $1 / 3$, combined with a puncturing process and an interleaver to have an overall coding rate of $1 / 2$. Results are given for 6 iterations at the decoder, and show that the global system benefits from the spatial diversity. In MISO case, it confirms that a simple STBC with unitary-rate equal to the channel rank, while coding is performed at bit level with the channel coder, is a good trade-off between complexity and performance for realistic scenarios. We also observe a gain of roughly $2 \mathrm{~dB}$ for a BER $=10^{-5}$ in favor of MIMO MMSE compared to MISO MMSE. 


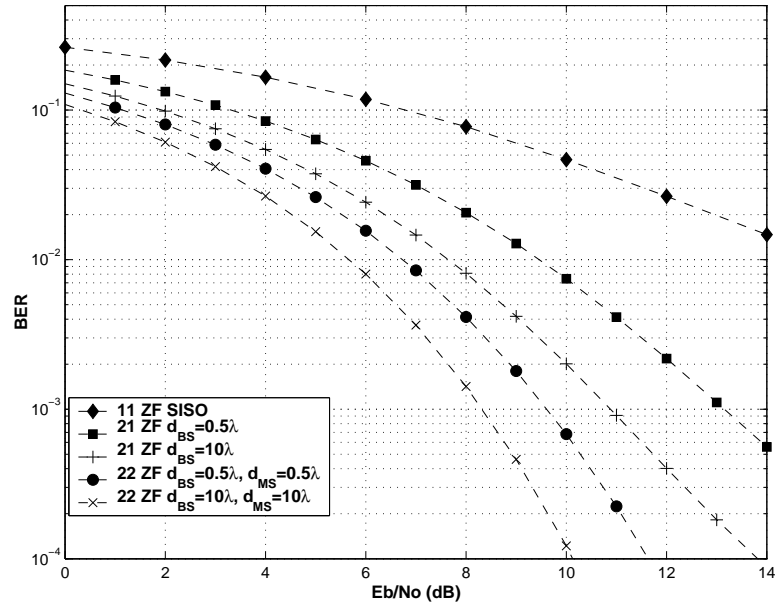

Fig. 4. Performance without channel coding of ZF $\left(L_{c}=N_{u}=32\right)$ over outdoor channels for SISO, MISO and MIMO systems.

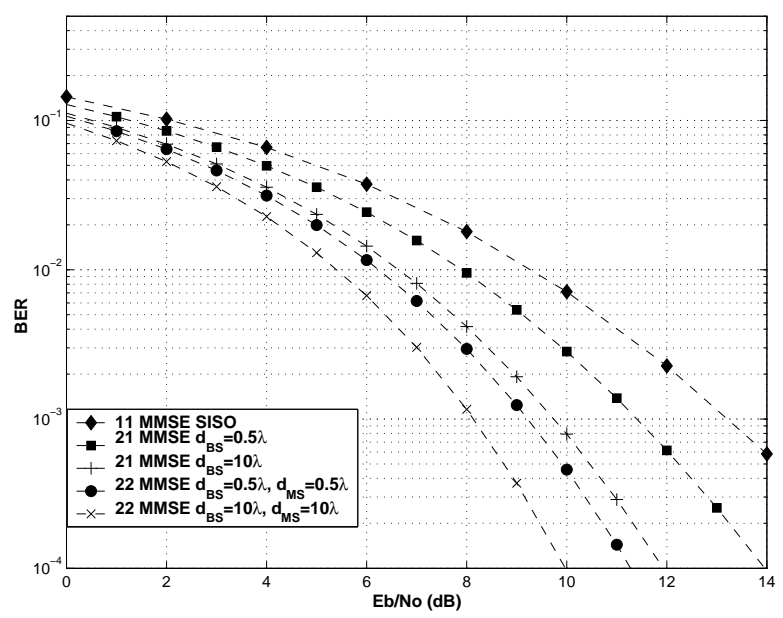

Fig. 5. Performance without channel coding of MMSE at full load $\left(L_{c}=\right.$ $N_{u}=32$ ) over outdoor channels for SISO, MISO, and MIMO systems.

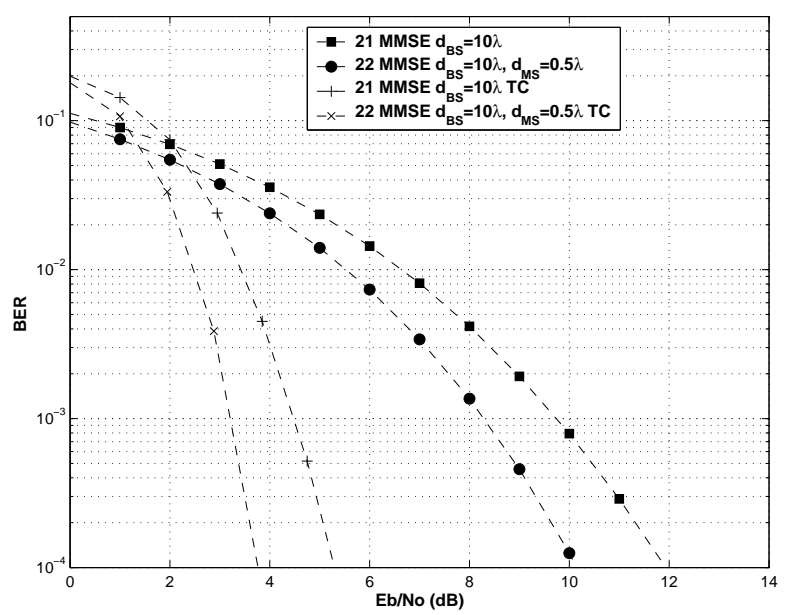

Fig. 6. Performance with UMTS-like turbo coding of MMSE at full-load ( $\left.L_{c}=N_{u}=32\right)$ over outdoor channels for MISO and MIMO systems.

\section{CONCLUSION}

The performance of ZF and MMSE Single-user Detection in orthogonal STBC MC-CDMA systems has been compared over realistic MIMO channel models in the case of two transmit antennas and one or two receive antennas. Two different MCCDMA systems have been considered for indoor and outdoor scenarios. The different results obtained as well for indoor scenarios as for outdoor scenarios demonstrate that spatial diversity improves significantly the performance of MC-CDMA systems. Then, Alamouti's STBC MC-CDMA scheme derives full benefit from the frequency and spatial diversities and can be considered as a very realistic and promising candidate for the air interface downlink of the 4th generation mobile radio systems.

\section{ACKNOWLEDGMENT}

The work presented in this paper was partly supported by the European FP5 IST project MATRICE (Multicarrier-CDMA TRansmission Techniques for Integrated Broadband CEllular Systems - http: //www.ist-matrice.org).

\section{REFERENCES}

[1] S. Hara and R. Prasad, "Overview of multicarrier CDMA", in IEEE Communications Magazine, vol. 35, no. 12, pp. 126-133, Dec. 1997.

[2] N. Yee, J.-P. Linnartz, and G. Fettweis, "Multi-carrier CDMA in indoor wireless radio networks", in IEEE Personal, Indoor and Mobile Radio Communications Symposium, Sept. 1993, pp. 109-113.

[3] K. Fazel and L. Papke, "On the performance of convolutionally-coded CDMA/OFDM for mobile communication system", in IEEE Personal, Indoor and Mobile Radio Communications Symposium, Sept. 1993, pp. 468-472.

[4] A. Chouly, A. Brajal, and S. Jourdan, "Orthogonal multicarrier techniques applied to direct sequence spread spectrum CDMA systems", in IEEE Global Communications Conference, Nov. 1993, pp. 1723-1728.

[5] V. DaSilva and E. Sousa, "Performance of orthogonal CDMA codes for quasi-synchronous communications systems", in Second International Conference on Universal Personal Communications, Oct. 1993, pp. 995999.

[6] M. Hélard, R. LE Gouable, J.-F. Hélard, and J.-Y. Baudais, "Multicarrier CDMA for future wideband wireless networks", in Annales des télécommunications, vol. 56, no. 5/6, pp. 260-274, May/June 2001.

[7] J.-Y. Baudais, J.-F. Hélard, and J. Citerne, "An improved linear MMSE detection technique for multi-carrier CDMA system: Comparison and combination with interference cancellation schemes", in European Transactions on Communications, vol. 11, no. 7, pp. 547-554, Nov./Dec. 2000.

[8] S. Alamouti, "A simple transmit diversity technique for wireless communications", in IEEE Journal on Selected Area in Communications, vol. 16, no. 8, pp. 1451-1458, Oct. 1998.

[9] V. Tarokh, H. Jafarkhani, and A. Calderbank, "Space-time block coding for wireless communications: Performance results", in IEEE Journal on Selected Area in Communications, vol. 17, no. 3, pp. 451-460, Mar. 1999.

[10] V. Le Nir, J.-M. Auffray, M. Hélard, J.-F. Hélard, and R. Le Gouable, "Combination of space-time block coding with MC-CDMA technique for MIMO systems with two, three and four transmit antennas", in IST Mobile \& Wireless Communication Summit, Aveiro, Portugal, June 2003.

[11] K. Pedersen, J. Andersen, J. Kermoal, and P. Mogensen, "A stochastic multiple input multiple output radio channel model for evaluation of space-time coding algorithms", in IEEE Vehicular Technology Conference, Sept. 2000.

[12] 3GPP2 TR 25.996 2003, "Network Spatial Model for Multiple Input Multiple Output Simulations (Release 6)", in 3GPP, Spatial channel AHG, SCM-134, Apr. 2003. 